\title{
Correction to: "Comparative repair capacity of knee osteochondral defects using regenerated silk fiber scaffolds and fibrin glue with/without autologous chondrocyes during 36 weeks in rabbit model
}

\author{
Somaieh Kazemnejad ${ }^{1}$ - Manijeh Khanmohammadi ${ }^{1}$ - Sahba Mobini ${ }^{1}$ - Masoud Taghizadeh-Jahed ${ }^{1}$. \\ Sayeh Khanjani ${ }^{1}$ - Shaghayegh Arasteh ${ }^{1} \cdot$ Hannaneh Golshahi $^{2} \cdot$ Giti Torkaman $^{3}$ - Roya Ravanbod ${ }^{3}$. \\ Hamed Heidari-Vala ${ }^{1}$ - Ali Moshiri ${ }^{4} \cdot$ Mohammad-Naghi Tahmasebi $^{5} \cdot$ Mohammad-Mehdi Akhondi $^{1}$
}

Published online: 27 May 2019

(C) Springer-Verlag GmbH Germany, part of Springer Nature 2019

\section{Correction to: Cell Tissue Res (2016) 364:559-572 https://doi.org/10.1007/s00441-015-2355-9}

In this paper, figure 1 and its associated text were erroneously identical to that of another article from our group (Mobini et al., 2016, Journal of Biomaterial Application, SAGE publications). Unfortunately, copyright permission to re-use figure 1 and its related data were not requested. The authors would like to apologize for any confusion caused in this regard.

Now, copyright permission for using the figure in this article was obtained from SAGE Publications, Inc as authentic holder of this image and we made the following corrigendum in the text:

The online version of the original article can be found at https://doi.org/ 10.1007/s00441-015-2355-9

Somaieh Kazemnejad

s.kazemnejad@avicenna.ac.ir; kazemnejad_s@yahoo.com

1 Reproductive Biotechnology Research Center, Avicenna Research Institute, Academic Center for Education, Culture and Research (ACECR), P.O. Box: 19615-1177, Tehran, Iran

2 Department of Pathology, Faculty of Veterinary Medicine, University of Tehran, Tehran, Iran

3 Department of Physical Therapy, Faculty of Medical Sciences, Tarbiat Modares University, Tehran, Iran

4 Razi Drug Research Center, Iran University of Medical Sciences, Tehran, Iran

5 Orthopedic Department, Shariati Hospital, Tehran University of Medical Sciences, Tehran, Iran
This phrase has been added to the end of figure 1 legend: "Adapted with permission from Mobini et al., 2016. Copyright (C) 2019 SAGE Publications, Inc"

Section 2.4 has to be corrected as follows:

Subcutaneous implantation of the scaffolds

To assess biodegradability and biocompatibility, the scaffolds (RS and RSF 2\%wt) were implanted subcutaneously in C57BL/6 mice and monitored during 90 days according to our previous paper (Mobini et al., 2016). In brief, under anaesthetization and aseptic condition, four longitudinal skin incisions (5 mm length) were made on the dorsum back of nine C57BL/6 mice and then two types of scaffolds in diskshaped $(3 \times 4 \mathrm{~mm})$ were implanted subcutaneously in each animal. Postoperative analgesia (tramadol;10 mg/Kg twice a day for three days) and antibiotic (Gentamicin; $10 \mathrm{mg} / \mathrm{Kg}$ of body weight daily for five days) were provided by intra muscular administration. 30-90 days after implantation, the animals were euthanized to remove the scaffolds for assessment. Each scaffold was considered as an internal control for the other scaffolds.

Section 3.1 has to be corrected as follows:

Gross and histopathological findings of subcutaneous implanted scaffolds in C57BL/6 mice

At day 30 post implantation, the RS $2 \%$ wt scaffolds were covered by a loose thin capsule. Moreover, a number of inflammatory cells penetrated the scaffold, adhered to its pores and no evidence of connective tissue regeneration was observed at this stage. The internal architecture was devoid of cellular structures at 60 days post implantation. However, the 
thickness of the capsule slightly raised and few blood vessels were observed in the covering capsule. At 90 days post implantation, a thick capsule was evident around RS $2 \%$ wt, however, the architecture of the scaffold was preserved and only a few amount of regeneration occurred. In contrast to the RS $2 \%$ wt scaffold, the RSF $2 \%$ wt scaffold was degraded and substituted by the new connective tissue 30 days post implantation. Only a few amounts of degummed silk fibers remained in which their diameters gradually extenuated. The covering capsule properly integrated with the regenerated connective tissue inside the scaffold. After 90 days, small amount of capsule remained and it mostly incorporated with the scaffold. In a comparative examination, the RSF $2 \%$ wt scaffold indicated greater percentage of filled pores, and typically lower amount of the remained pores in comparison with the RS $2 \%$ wt scaffold. Moreover, compared to the RS $2 \%$ wt scaf- folds, the RSF $2 \%$ wt scaffolds indicated significantly higher number of fibroblasts, fibrocytes and total vessels, and lower number of macrophages (Fig.1).

As a new citation the following reference has to be added:

Mobini S, Taghizadeh-Jahed M, Khanmohammadi M, Moshiri A, Naderi MM, Heidari-Vala H, Ashrafi Helan J, Khanjani S, Springer A, Akhondi MM, Kazemnejad S. Comparative evaluation of in vivo biocompatibility and biodegradability of regenerated silk scaffolds reinforced with/ without natural silk fibers." Journal of biomaterials applications 30.6 (2016): 793- 809

Publisher's note Springer Nature remains neutral with regard to jurisdictional claims in published maps and institutional affiliations. 